\title{
Exploring Whether Super-puffs can be Explained as Ringed Exoplanets
}

\author{
Anthony L. Piro ${ }^{1}$ (D) and Shreyas Vissapragada ${ }^{2}$ (iD \\ ${ }^{1}$ The Observatories of the Carnegie Institution for Science, 813 Santa Barbara St., Pasadena, CA 91101, USA; piro@ carnegiescience.edu \\ ${ }^{2}$ Division of Geological and Planetary Sciences, California Institute of Technology, 1200 E. California Blvd., Pasadena, CA 91125, USA; svissapr@caltech.edu \\ Received 2019 November 26; revised 2020 January 28; accepted 2020 January 28; published 2020 February 28
}

\begin{abstract}
An intriguing, growing class of planets are the "super-puffs," objects with exceptionally large radii for their masses and thus correspondingly low densities $\left(\lesssim 0.3 \mathrm{~g} \mathrm{~cm}^{-3}\right)$. Here we consider whether they could have large inferred radii because they are in fact ringed. This would naturally explain why super-puffs have thus far only shown featureless transit spectra. We find that this hypothesis can work in some cases but not all. The close proximity of the super-puffs to their parent stars necessitates rings with a rocky rather than icy composition. This limits the radius of the rings, and makes it challenging to explain the large size of Kepler 51b, 51c, 51d, and 79d unless the rings are composed of porous material. Furthermore, the short tidal locking timescales for Kepler 18d, 223d, and 223e mean that these planets may be spinning too slowly, resulting in a small oblateness and rings that are warped by their parent star. Kepler 87c and 177c have the best chance of being explained by rings. Using transit simulations, we show that testing this hypothesis requires photometry with a precision of somewhere between $\sim 10 \mathrm{ppm}$ and $\sim 50 \mathrm{ppm}$, which roughly scales with the ratio of the planet and star's radii. We conclude with a note about the recently discovered super-puff HIP 41378f.
\end{abstract}

Unified Astronomy Thesaurus concepts: Occultation (1148); Exoplanets (498); Exoplanet rings (494); Exoplanet detection methods (489)

\section{Introduction}

In our solar system, rings are common among the four outer gas-rich planets as well as present for some of the smaller rocky bodies. Nevertheless, discovering rings around planets outside our solar systems has been challenging. In principle, rings should be detectable from detailed photometric or spectroscopic changes to transits (e.g., Barnes \& Fortney 2004; Ohta et al. 2009; Santos et al. 2015; Zuluaga 2015; Akinsanmi et al. 2018). The difficulty is that such signals are subtle and difficult to discern in current data. In a few cases, potential rings or constraints on rings have been made in this way (Heising et al. 2015; Aizawa et al. 2017, 2018), and in at least one instance it has been argued that an exoplanet has a giant ring system from a series of complex eclipses (Kenworthy \& Mamajek 2015; Rieder \& Kenworthy 2016). There is clearly still a lot we do not know about the rings of exoplanets.

The simplest impact of rings is to increase the depth of transits so that instead of measuring the planet radius $R_{p}$, an eclipsed area of $A$ results in an inferred radius of (Piro 2018a)

$$
R_{\text {inf }}=(A / \pi)^{1 / 2} \gtrsim R_{p} .
$$

A useful example to consider is that of Saturn: averaged over season, if an external observer measured Saturn's size in transit without accounting for rings, they would underestimate its true density by about a factor of two. Thus if a population of exoplanets are found with anomalously large radii, and correspondingly low densities, this may indicate we are observing $R_{\text {inf }}$ rather than $R_{p}$.

In fact, there is a growing class of exoplanets with inferred densities of $\lesssim 0.3 \mathrm{~g} \mathrm{~cm}^{-3}$, also known as "super-puffs" (Cochran et al. 2011; Jontof-Hutter et al. 2014; Masuda 2014; Ofir et al. 2014; Mills et al. 2016; Santerne et al. 2019; Vissapragada et al. 2020). The properties of these planets and their host stars are summarized in Table 1. We note that different authors have differing definitions for this class of planets, with some adopting a strict boundary of $M_{p}<10 M_{\oplus}$ (e.g., Jontof-
Hutter 2019; Lee 2019). Here we take the slightly more liberal approach of including planets with $M_{p} \lesssim 15 M_{\oplus}$, which includes the low-density planets Kepler-18d (Cochran et al. 2011) and Kepler-177c (Vissapragada et al. 2020).

This new class of planets with larger radii than expected bears some similarity to the classical problem of hot-Jupiter radius inflation. However, hot-Jupiter inflation is strongly correlated with equilibrium temperature (Miller \& Fortney 2011; Thorngren \& Fortney 2018), which means that a similar mechanism cannot be extended to the much cooler super-puffs. While some super-puff systems are young, and therefore may appear inflated because they are still contracting (LibbyRoberts et al. 2020), most of these planets are older and cannot be explained with youth either. Other proposed explanations for these planets include dusty outflows (Wang \& Dai 2019), photochemical hazes (Kawashima et al. 2019), inflation from tidal heating (Millholland 2019), or especially thick gas envelopes (Lee \& Chiang 2016). If it is the latter, then these exoplanets would be prime targets for transit spectroscopy, but when this has been performed the results are featureless spectra (Libby-Roberts et al. 2020).

Here we consider the alternative hypothesis that super-puffs are in fact ringed exoplanets. In Section 2, we explore whether super-puffs can be explained as planets with rings, and what this implies about both the rings and the underlying planets. We find that this explanation works for some of the superpuffs, but for others it has difficulties. In Section 3, we perform transit simulations to assess whether this hypothesis can be constrained by current or future observational efforts. We then conclude in Section 4 with a summary of this work.

\section{Constraints from the Ring Hypothesis}

We first consider the hypothesis that super-puffs are actually planets with rings, and investigate what this implies about the properties of such rings and the planets themselves. 
Table 1

Super-puff Planet and Parent Star Properties

\begin{tabular}{lcccccccccc}
\hline \hline Name & $M_{p}\left(M_{\oplus}\right)$ & $R_{\text {inf }}\left(R_{\oplus}\right)$ & $\langle\rho\rangle\left(\mathrm{g} \mathrm{cm}^{-3}\right)$ & $a(\mathrm{au})$ & $P($ days $)$ & $M_{*}\left(M_{\odot}\right)$ & $R_{*}\left(R_{\odot}\right)$ & $T_{\text {eff }}(\mathrm{K})$ & $\left(R_{\text {inf }} / R_{*}\right)^{2}$ & Reference \\
\hline Kepler 18d & 16.4 & 6.98 & 0.27 & 0.12 & 14.86 & 0.97 & 1.11 & 5345 & $3.3 \times 10^{-3}$ & $(1)$ \\
Kepler 51b & 3.7 & 6.89 & 0.06 & 0.25 & 45.15 & 0.99 & 0.88 & 5670 & $5.1 \times 10^{-3}$ & $(2,3)$ \\
Kepler 51c & 4.4 & 8.98 & 0.03 & 0.38 & 85.31 & $\ldots$ & $\ldots$ & $\ldots$ & $8.7 \times 10^{-3}$ & $\ldots$ \\
Kepler 51d & 5.7 & 9.46 & 0.04 & 0.51 & 130.18 & $\ldots$ & $\ldots$ & $\ldots$ & $9.7 \times 10^{-3}$ & $\ldots$ \\
Kepler 79d & 6.0 & 7.16 & 0.09 & 0.29 & 52.09 & 1.17 & 1.30 & 6174 & $2.5 \times 10^{-3}$ & $(4)$ \\
Kepler 87c & 6.4 & 6.14 & 0.15 & 0.68 & 191.23 & 1.10 & 1.82 & 5600 & $9.6 \times 10^{-4}$ & $(5)$ \\
Kepler 177c & 14.7 & 8.73 & 0.12 & 0.26 & 49.41 & 0.92 & 1.32 & 5732 & $3.6 \times 10^{-3}$ & $(6)$ \\
Kepler 223d & 8.0 & 5.24 & 0.31 & 0.13 & 14.79 & 1.13 & 1.72 & 5821 & $7.8 \times 10^{-4}$ & $(7)$ \\
Kepler 223e & 4.8 & 4.60 & 0.28 & 0.15 & 19.73 & $\ldots$ & $\ldots$ & $\ldots$ & $6.0 \times 10^{-4}$ \\
HIP 41378f & 12 & 9.2 & 0.09 & 1.37 & 542.08 & 1.16 & 1.27 & 6320 & $4.4 \times 10^{-3}$ & $(8)$ \\
\hline
\end{tabular}

Note. (1) Cochran et al. (2011), (2) Masuda (2014), (3) Libby-Roberts et al. (2020), (4) Jontof-Hutter et al. (2014), (5) Ofir et al. (2014), (6) Vissapragada et al. (2020), (7) Mills et al. (2016), (8) Santerne et al. (2019).

\subsection{Constraints on Ring Material}

The rings of Saturn would be the closest analog to what we are considering here, since super-puff rings must be extended and optically thick if they are to cause such large inferred radii. An important difference in comparison to Saturn is that superpuffs are much closer to their parent stars. Ice sublimates at a temperature of $T_{\text {sub }} \approx 170 \mathrm{~K}$, so rings cannot be composed of ice for planets with a semimajor axis within (Gaudi et al. 2003)

$$
a \lesssim\left(\frac{L_{*}}{16 \pi \sigma_{\mathrm{SB}} T_{\mathrm{sub}}^{4}}\right)^{1 / 2} \approx 2.7\left(\frac{L_{*}}{L_{\odot}}\right)^{1 / 2} \text { au. }
$$

For this reason, the material forming the rings of super-puffs will be rocky with a typical density in the range of $\approx 2-5 \mathrm{~g} \mathrm{~cm}^{-3}$, depending on the exact composition. This is only approximate, and may extend somewhat below $2 \mathrm{~g} \mathrm{~cm}^{-3}$ for material with a higher porosity.

Rings viscously spread until the outer edge reaches the fluid Roche limit (Murray \& Dermott 1999),

$$
R_{r} \approx 2.46 R_{p}\left(\frac{\rho_{p}}{\rho_{r}}\right)^{1 / 3},
$$

where $\rho_{p}$ is the bulk density of the planet and $\rho_{r}$ is the density of particles that make up the rings. Outside of this radius, material aggregates into satellites and is no longer part of the ring. At most, the rings can cover an area of $f \pi R_{r}^{2}$, where $f \lesssim 1$ is the filling factor that accounts for gaps and rings with optical depth less than unity (as is well known for Saturn). The observed inferred radii then obey $R_{\text {inf }} \lesssim f^{1 / 2} R_{r}$, because the exoplanet may be viewed at an oblique angle. Combining this with Equation (3), we put a limit on the ring density,

$$
\begin{aligned}
\rho_{r} & \lesssim\left(\frac{2.46 f^{1 / 2}}{R_{\text {inf }}}\right)^{3} \frac{3 M_{p}}{4 \pi} \\
& \approx 2.3 f^{3 / 2}\left(\frac{M_{p}}{6 M_{\oplus}}\right)\left(\frac{R_{\text {inf }}}{6 R_{\oplus}}\right)^{-3} \mathrm{~g} \mathrm{~cm}^{-3} .
\end{aligned}
$$

Material with a density above this will not be able to make sufficiently large rings because it combines into satellites at large radii instead. In Figure 1, we plot this density for each of the super-puffs. We include error bars, which correspond to the current uncertainties in the mass and inferred radius for each

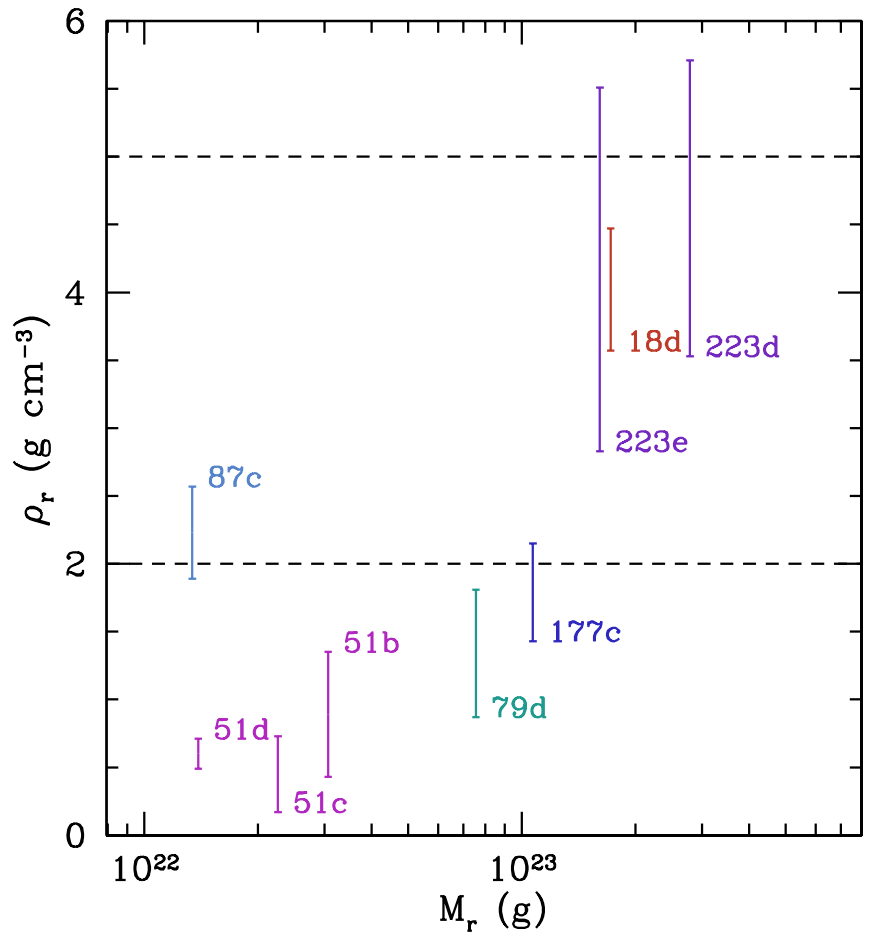

Figure 1. Ring material density $\rho_{r}$ and mass $M_{r}$ constraints if super-puffs were instead ringed using Equations (4) and (6), respectively. Super-puffs that lie between the two horizontal dashed lines can potentially have their large radii explained as rocky rings. Those below the lower dashed line have difficulty being explained in this way unless the ring material is more porous. The ring mass is required for the ring to last a timescale of $t_{\mathrm{PR}}=10^{9} \mathrm{yr}$ with an inclination angle of $i=45^{\circ}$. The range of ring masses needed is comparable to large asteroids in our solar system.

planet, and take $f=1$ since even smaller $\rho_{r}$ values are required for $f<1$. The dashed horizontal lines roughly delineate the density range expected for rocky material. From this comparison, we see that Kepler 18d, 87c, 223d, and 223e could all be explained by rocky rings, while Kepler $177 \mathrm{c}$ is borderline. On the other hand, Kepler 51b, 51c, 51d, and 79d are so large that it is difficult to explain them with rocky rings unless the material is very porous (although not out of the question, since some asteroids have densities as low as $\sim 1.5 \mathrm{~g} \mathrm{~cm}^{-3}$; Carry 2012). It has been argued that the locations of the solar system rings might indicate that weak material that can easily be disrupted is required for generating rings (Hedman 2015). 
Although very speculative, this may explain the low densities we infer for the super-puff ring material here.

Another constraint is that the rings will be subject to Poynting-Robertson drag because of the relative close proximity to their parent stars (Goldreich \& Tremaine 1978). Since the rings need to be optically thick to produce the large observed transits, the corresponding Poynting-Robertson timescale depends on the mass surface density $\Sigma$ (rather than the particle size as is the case for typical Poynting-Robertson drag; Schlichting \& Chang 2011), resulting in

$$
t_{\mathrm{PR}} \approx \frac{\pi \sum c^{2}}{\sin i\left(5+\cos ^{2} i\right)} \frac{4 \pi a^{2}}{L_{*}},
$$

where $i$ is the inclination of the ring with respect to the orbital plane and $L_{*}$ is the luminosity of the parent star. The total ring mass is roughly $M_{r} \approx 3 \pi f \Sigma R_{r}^{2} / 4$ (Piro 2018a). Using $R_{\text {inf }} \lesssim R_{r}$, we can at least get a limit on the ring mass needed if the rings are to last a time $t_{\mathrm{PR}}$,

$$
M_{r} \approx \frac{3 t_{\mathrm{PR}}}{4 c^{2}} \frac{f L_{*}}{4 \pi a^{2}} R_{\mathrm{inf}}^{2} \sin i\left(5+\cos ^{2} i\right) .
$$

This mass is plotted for each of the super-puffs in Figure 1 for a timescale of $t_{\mathrm{PR}}=10^{9} \mathrm{yr}$ and an inclination of $i=45^{\circ}$ (again with $f=1$, which gives an upper limit). The range of masses is similar to large asteroid masses in our solar system (Lang 1992), showing that these are not unreasonable amounts of material for rings.

We note that another important timescale to consider is the viscous time for the rings, which should be dominated by collisions including self-gravity effects (see discussions in Daisaka et al. 2001; Piro 2018a). In detail, this depends on the exact density and size of the ring particles, but can easily be in the range of $\sim 10^{8}-10^{9} \mathrm{yr}$.

\subsection{Constraints on the Planetary Quadrupole Moment}

The presence of rocky rings with the desired properties also provides constraints for the underlying planets, which we explore in more detail next.

Another issue is that the rings must be oriented at an oblique angle with respect to the planet's orbital plane to produce the large transits. The ring's orientation depends on the competing effects of the planet's oblateness, quantified by the quadrupole gravitational harmonic $J_{2}$, and the tide from the parent star (Tremaine et al. 2009). Equating these two effects provides an estimation of the so-called Laplace radius (Schlichting \& Chang 2011),

$$
R_{\mathrm{L}}^{5} \approx 2 J_{2} M_{p} R_{p}^{2} \frac{a^{3}}{M_{*}}
$$

where we have assumed that the orbital eccentricity is negligible (justified by what is known for these multiplanet systems; Fabrycky et al. 2014; Hadden \& Lithwick 2014, 2017). Beyond this radius, a ring is warped into the orbital plane of the planet. Thus, we require $R_{L} \gtrsim R_{r}$ for the super-puffs. This implies a minimum $J_{2}$ of

$$
J_{2}\left(R_{\mathrm{L}}=R_{r}\right) \approx \frac{(2.46)^{2} R_{\text {inf }}^{3}}{2 a^{3}}\left(\frac{M_{*}}{M_{p}}\right)\left(\frac{\rho_{p}}{\rho_{r}}\right)^{2 / 3},
$$

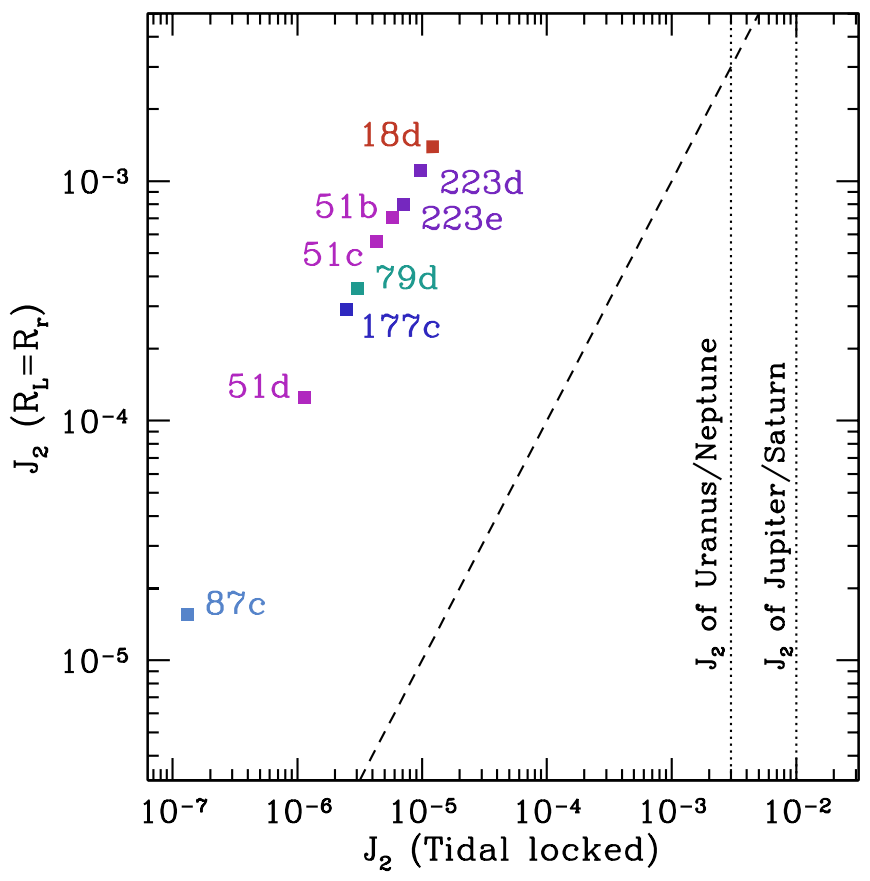

Figure 2. Comparison of the two values for $J_{2}$ from Equations (8) and (10). The diagonal dashed line shows where these two quantities are equal. Since all the super-puffs are to the left of this line, they are spinning too slowly and would have rings warped into their orbital planes if they are tidally locked. On the other hand, if the super-puffs have spins similar to the gas or ice giants in our solar system, and if they are able to prevent tidal locking, then their rings would not be warped.

where we first use Equation (3) to substitute $R_{r}$ for $R_{p}$ (since the radius of the underlying planet is unknown), and then we use $R_{\text {inf }} \lesssim R_{r}$ to estimate $J_{2}$ from the current observables.

The actual $J_{2}$ of a super-puff depends on its rotation rate $\Omega$. This can be estimated as (Chandrasekhar 1969)

$$
J_{2} \approx \Lambda \frac{\Omega^{2} R_{p}^{3}}{G M_{p}},
$$

where $\Lambda \approx 0.2-0.5$ is a factor that depends on the density distribution of the planet. Given the close proximity of these planets to their parent stars, it is natural to assume they are tidally locked. This would result in

$$
\begin{aligned}
J_{2} \text { (Tidal locked) } & \approx \Lambda\left(\frac{M_{*}}{M_{p}}\right)\left(\frac{R_{p}}{a}\right)^{3} \\
& \approx 0.02\left(\frac{\Lambda}{0.3}\right)\left(\frac{M_{*}}{M_{p}}\right)\left(\frac{R_{\text {inf }}}{a}\right)^{3}\left(\frac{\rho_{r}}{\rho_{p}}\right),
\end{aligned}
$$

where we again use Equation (3) and $R_{\text {inf }} \lesssim R_{r}$ to write this in

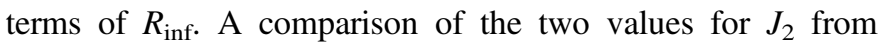
Equations (8) and (10) is plotted in Figure 2. We use $\rho_{r} / \rho_{p} \sim 2$, corresponding to a rocky ring composition. In all cases, the $J_{2}$ implied for tidal locking is less than the $J_{2}$ needed to prevent ring warping. Therefore, if the super-puffs are tidally locked, then none of them can have rings at the inclinations needed to produce the large inferred radii.

On the other hand, the current $J_{2}$ of the gas and ice giants in our solar system are much larger with values of $\sim 0.003-0.01$ as indicated on Figure 2. If the super-puffs could have similar 


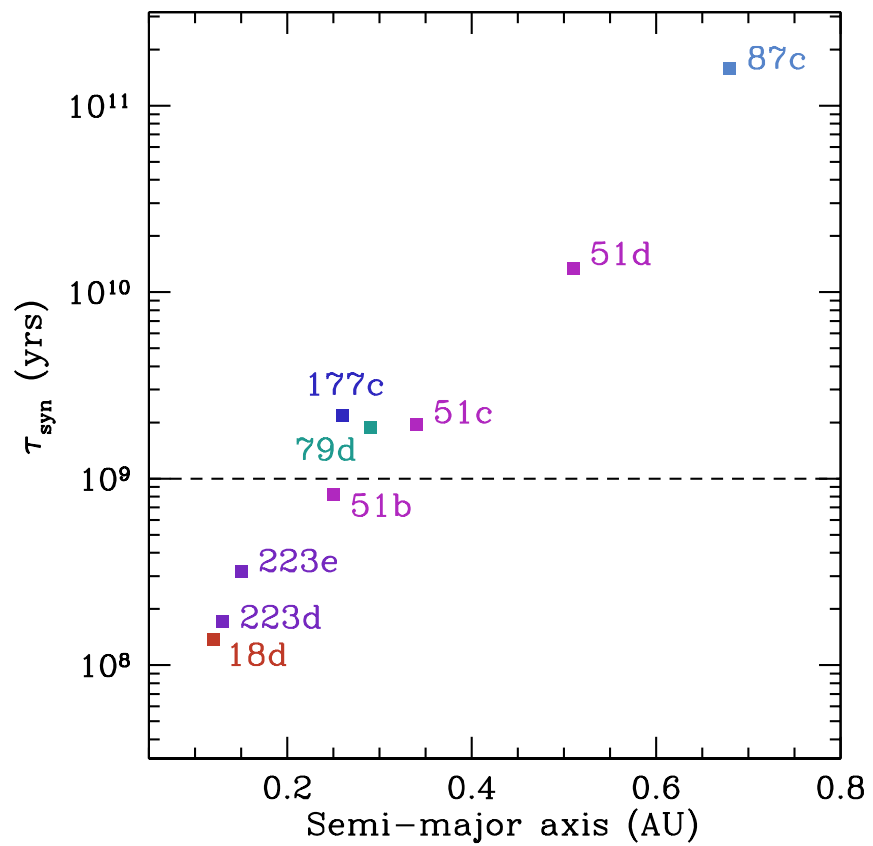

Figure 3. Synchronization time $\tau_{\mathrm{syn}}$ for each of the super-puffs as a function of their semimajor axis $a$ for $Q_{p}=10^{6.5}$ and $k_{2}=3 / 2$. The dashed line delineates $10^{9} \mathrm{yr}$. Planets above this line may be expected to maintain a $J_{2}$ independent of tidal locking.

$J_{2}$ values, and not be impacted too drastically by tidal locking, then they could still have sufficiently large $J_{2}$ to prevent their rings from being warped.

Motivated by this, we consider the tidal synchronization time for each super-puff, roughly estimated as (Piro 2018b)

$$
\tau_{\text {syn }} \approx \frac{2 \lambda Q_{p}}{3 k_{p}}\left(\frac{M_{p}}{M_{*}}\right)\left(\frac{a}{R_{p}}\right)^{3} \frac{P}{2 \pi},
$$

where $\lambda \approx 0.2-0.3$ is the radius of gyration, $Q_{p}$ is the tidal quality factor, and $k_{p}$ is the Love number. The resulting $\tau_{\text {syn }}$ for each super-puff is summarized in Figure 3, plotted using similar estimates as above for $R_{p}$, and with $\lambda=0.3, Q_{p}=$ $10^{6.5}$, and $k_{p}=3 / 2$. This shows that the tidal locking timescale is mostly a function of the semimajor axis, which is not surprising since $\tau_{\text {syn }} \propto a^{9 / 2}$. The super-puffs with $\tau_{\text {syn }} \gtrsim$ $10^{9} \mathrm{yr}$ may be able to maintain a sufficiently large $J_{2}$ to prevent ring warping. In contrast, Kepler 18d, 51b, 223d, and 223 e may become tidally locked and have a smaller $J_{2}$. Even in these cases though $\tau_{\text {syn }} \gtrsim 10^{9} \mathrm{yr}$, and these objects may still not be completely tidally locked if they are especially young (such as for Kepler 51; Libby-Roberts et al. 2020) or if there is a factor of a few underestimate of the synchronization time. If the value of $Q_{p}$ was taken to be much smaller (for example, rocky planets like Earth have $Q_{p} \sim 10$ ), then all of the superpuffs would be tidally locked. Therefore, the super-puffs must have substantial gaseous envelopes even if they are explained by rings.

\section{Detectability}

If some of the super-puffs are actually ringed, then this can be revealed in the details of their transit light curves (e.g., Barnes \& Fortney 2004; Santos et al. 2015; Akinsanmi et al. 2018).

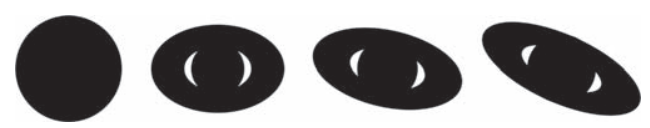

Figure 4. Example of a bare planet and three different ringed planets, each with the same covering area. From left to right, these are a bare planet with $R_{p}=6.98 R_{\oplus}$; a ringed planet with $R_{p}=3.5 R_{\oplus}, \theta=41.3, \phi=0^{\circ}$; a ringed planet with $R_{p}=4.0 R_{\oplus}, \theta=33^{\circ} .0, \phi=22.5$; and a ringed planet with $R_{p}=4.5 R_{\oplus}, \theta=34.1, \phi=45^{\circ}$.

Motivated by this, we simulate transits of ringed planets to assess whether this hypothesis is testable by current or future transit observations.

To construct the ringed planets, there are a few things to consider. First, the radius of the underlying planet $R_{p}$ must be chosen. This is unconstrained by the data, but must be in a range that gives a reasonable density given the planet's mass. Next, the inner and outer radius of the rings must be chosen. Since this depends on $\rho_{p}$ and $\rho_{r}$, both of which are unknown, we make the simple assumption that they extend from an inner radius of $\approx 1.25 R_{p}$ to $\approx 2.5 R_{p}$. Finally, we use the prescriptions summarized in Piro (2018a) to solve for the range of obliquities and "seasons" (the azimuthal angle of the planet) that together result in a transit depth that matches the observed $R_{\text {inf. }}$. An example of a bare planet and three different ring sizes and orientations is shown in Figure 4. This gives a sense for how a range of different silhouettes can provide the same maximum transit.

To perform these calculations, 5760 by 5760 pixel gray scale PNG images are generated for both the limb-darkened star and planet. For the limb-darkening prescription, we use the parameterization from Barnes \& Fortney (2003) with the coefficients $c_{1}=0.64$ and $c_{2}=-0.065$. These are simply chosen to mimic a realistic star for the examples presented here. In a true comparison to a specific super-puff, these coefficients should be fit for when modeling the transit. A useful feature of the super-puffs is that they are all in multitransiting systems. Therefore the limb-darkening can be measured from the normal radius planets to be used for the super-puff ring fitting. ${ }^{3} \mathrm{We}$ wrote a simple code to place the ringed planet at different locations across star, multiply the two images, and then sum up the pixels to find the total emitted light at any given time. These calculations do not include any forward scattering effects, since it has a relatively small impact in comparison to the many uncertainties for these systems. Since the strength of forward scattering depends on the size of the grains in the rings, its measurement may provide a more detailed understanding of the ring composition (Barnes \& Fortney 2004).

One of these calculations is presented in Figure 5. This example is a ringed planet with the same projected surface area as Kepler 18b. The planet radius is assumed to be $R_{p}=4.5 R_{\oplus}$ and the ring is positioned with obliquity $\theta=34^{\circ} .1$ and season $\phi=45^{\circ}$. The upper image shows the planet at seven different locations across the star's face. The middle and bottom panels show the resulting transit and the difference between a ringed transit and a bare transit with the same surface area, respectively. Each point indicates a time of one of the snapshots from the top image.

This example demonstrates how the tilted projection of the ring naturally results in an asymmetric light curve. At ingress (on the left side) the ringed planet begins covering the star

\footnotetext{
3 As an aside, an initial assessment on whether some super-puffs have rings may be possible by looking for differences in the limb-darkening fit to individual planets orbiting the same star (Akinsanmi et al. 2018).
} 

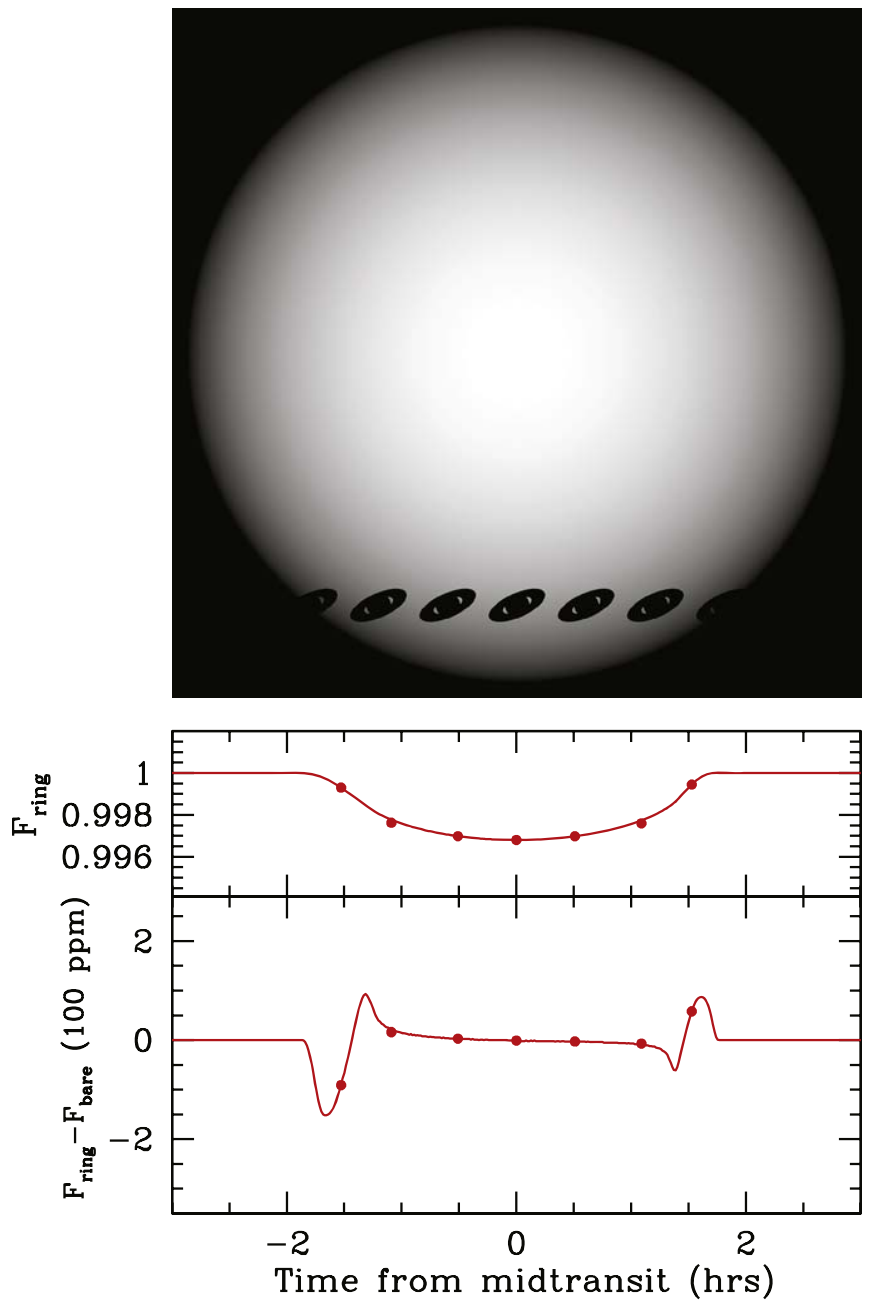

Figure 5. Transit of a ringed planet and the resulting light curves. The upper panel shows an example of the images considered for the transit calculations with the planet at seven different positions. The middle panel shows the resulting transit (normalized to the stellar flux) with the points corresponding to each of the planet positions shown in the upper panel. The bottom shows the difference between a ringed transit and a bare star with the same covering area.

earlier than the bare planet would, resulting in a deficit of light. Conversely, at egress (the right side), the ringed planet stops covering the star because it is nearly parallel with the stellar limb, while a bare planet would still block light. This results in additional light at late times.

In a more careful fit that compares bare and ringed transits, the stellar parameters should be fit in each case as well. This includes the stellar radius, limb darkening, and impact parameter of the planet. Here we use the values summarized in the literature from the fits assuming a bare planet for simplicity because our main goal is to qualitatively highlight how large the deviations are for ringed transits of super-puffs. A further complication when fitting super-puff transits is that they are all multiplanet systems, so the fits should account for both ringed and bare planets in the same systems to constrain the stellar parameters. Additionally, transit fits would also have to compare the evidence for a ringed planet model against that for an oblate planet model, as these two scenarios can produce similar transit shapes especially at high obliquity (Barnes \& Fortney 2003; Akinsanmi et al. 2018, 2019).

Beyond the complications with fitting ringed transits mentioned above, between the multitude of super-puffs, the

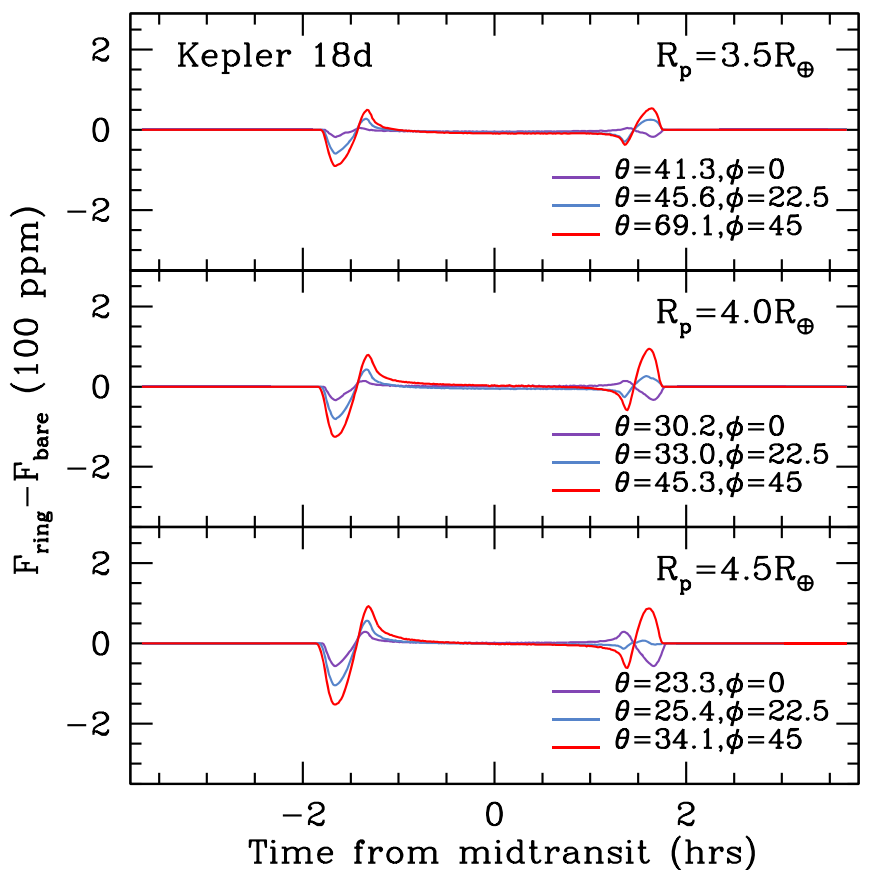

Figure 6. Sampling of possible ringed exoplanet models that would provide the same transit depth as Kepler 18d. The three panels use planet radii of $3.5 R_{\oplus}$, $4.0 R_{\oplus}$, and $4.5 R_{\oplus}$, from top to bottom. In each panel, three different combinations of angles are shown.

range of underlying planet radii that each could have, the range of ring radii depending on the ring composition, and the many possible viewing angles, there are a multitude of possible parameters to consider for ringed planet transits. For this reason, we focus on a few of the super-puffs as example cases and describe the general trends we have found.

\subsection{Kepler $18 d$}

The first super-puff we consider is Kepler 18d in Figure 6 (also highlighted previously in Figure 5). This is an example of a relatively high density super-puff that is also close to its host star. For these reasons, Kepler $18 \mathrm{~d}$ is attractive for having rocky rings because of the density required for its ring material, but less attractive because its relatively low synchronization timescale may mean that it is tidally locked to its host star and thus has a $J_{2}$ that is too low.

Figure 6 shows the results of our parameter survey for Kepler $18 \mathrm{~d}$. We consider radii of $3.5 R_{\oplus}, 4.0 R_{\oplus}$, and $4.5 R_{\oplus}$ for the underlying planet in the top, middle, and bottom panels, respectively. These correspond to mean densities for the planet of $2.1,1.4$, and $1.0 \mathrm{~g} \mathrm{~cm}^{-3}$, respectively, which are in the range expected for Saturn- or Neptune-like planets. We then construct rings with a viewing angle required to match the $R_{\text {inf }}$ measured for this super-puff. Three different seasons (values for the azimuthal angle $\phi$ ) are shown in each panel.

Some general trends can be seen across the nine models shown in Figure 6. As we vary the season, the silhouette of the ringed planet becomes more asymmetric, and the differences between the ringed and bare transits becomes correspondingly more asymmetric as well. Furthermore, the differences become larger when the underlying planet has a larger radius. This may seem paradoxical, but the reason is that when the planet is larger, the ring is viewed more edge on (as seen by the angle $\theta$ ). This makes the ring silhouette less like a large planet. 


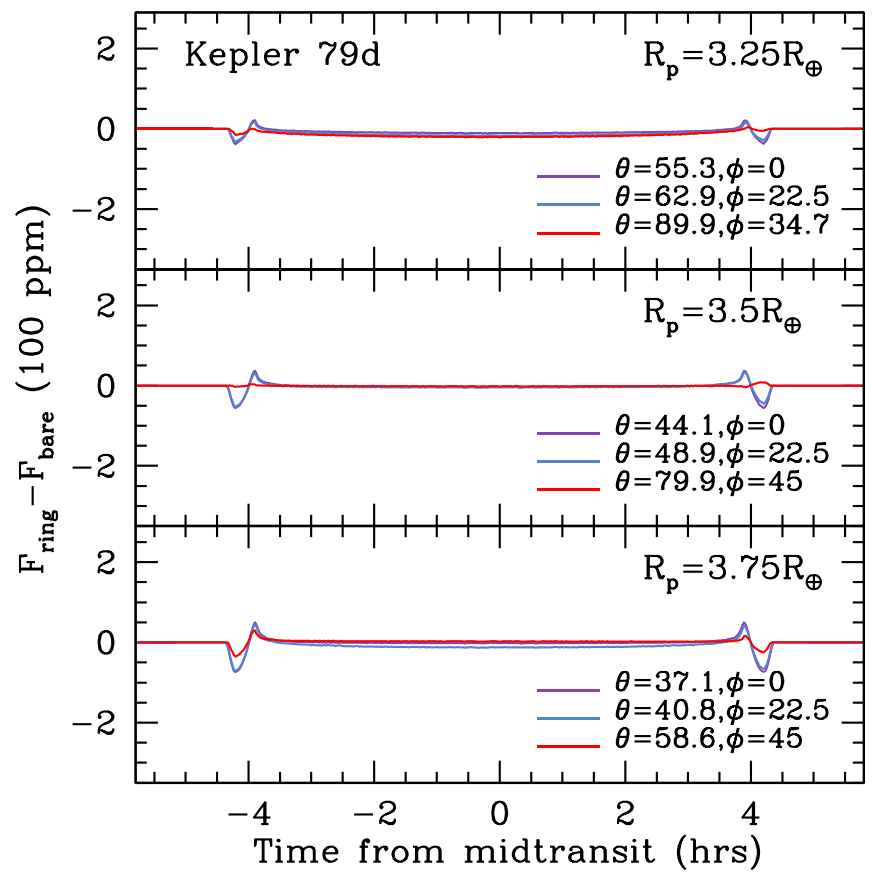

Figure 7. Same as for Figure 6, but for Kepler 79d.

\subsection{Kepler $79 d$}

Next, we consider Kepler 79d as an example of an especially low-density super-puff. The results are plotted in Figure 7. We again consider three different planet radii, but the range of potential radii are $3.25 R_{\oplus}, 3.5 R_{\oplus}$, and $3.75 R_{\oplus}$, which correspond to mean densities for the planet of $0.96,0.77$, and $0.67 \mathrm{~g} \mathrm{~cm}^{-3}$, respectively. This range is smaller because it is more difficult to match the observed especially large radius inferred for this exoplanet, even with rings. Furthermore, the obliquity of the planet must be relatively larger. This is because the ring must be viewed fairly face-on to cover enough area. Correspondingly, the transit is more like an oblate planet rather than a ring and thus the difference between the ring and bare cases is at most $\sim 50 \mathrm{ppm}$. This is smaller than it was for Kepler 18d, where the residual signal could exceed $\sim 150 \mathrm{ppm}$.

\subsection{Kepler $87 c$ and $177 c$}

Finally, we consider the cases of Kepler $87 \mathrm{c}$ and 177c. As discussed in Section 2, a combination of factors for these superpuffs make them some of the most attractive for being explained by rings: the required $\rho_{r}$ is in the range for rocky material, the $J_{2}$ required for $R_{\mathrm{L}} \gtrsim R_{r}$ is small so that the rings would likely not be warped, and the long synchronization times mean that they may be able to maintain this $J_{2}$.

The results for Kepler $87 \mathrm{c}$ and $177 \mathrm{c}$ are summarized in Figures 8 and 9, respectively. For Kepler 87c, we consider planetary radii of $3 R_{\oplus}, 3.4 R_{\oplus}$, and $3.8 R_{\oplus}$, which correspond to mean planet densities of $1.3,0.90$, and $0.64 \mathrm{~g} \mathrm{~cm}^{-3}$, respectively. The variations are found to be small for Kepler $87 \mathrm{c}$ because of the relatively small size of this planet with respect to its parent star, as shown by in Table 1 . Nevertheless, in the future photometry at a level of $\sim 10-30 \mathrm{ppm}$ one should be able to determine whether the ring hypothesis works or not for Kepler 87c. For Kepler 177c, we consider planetary radii of $4 R_{\oplus}, 4.5 R_{\oplus}$, and $5 R_{\oplus}$, which correspond to mean planet densities of 1.3, 0.89, and

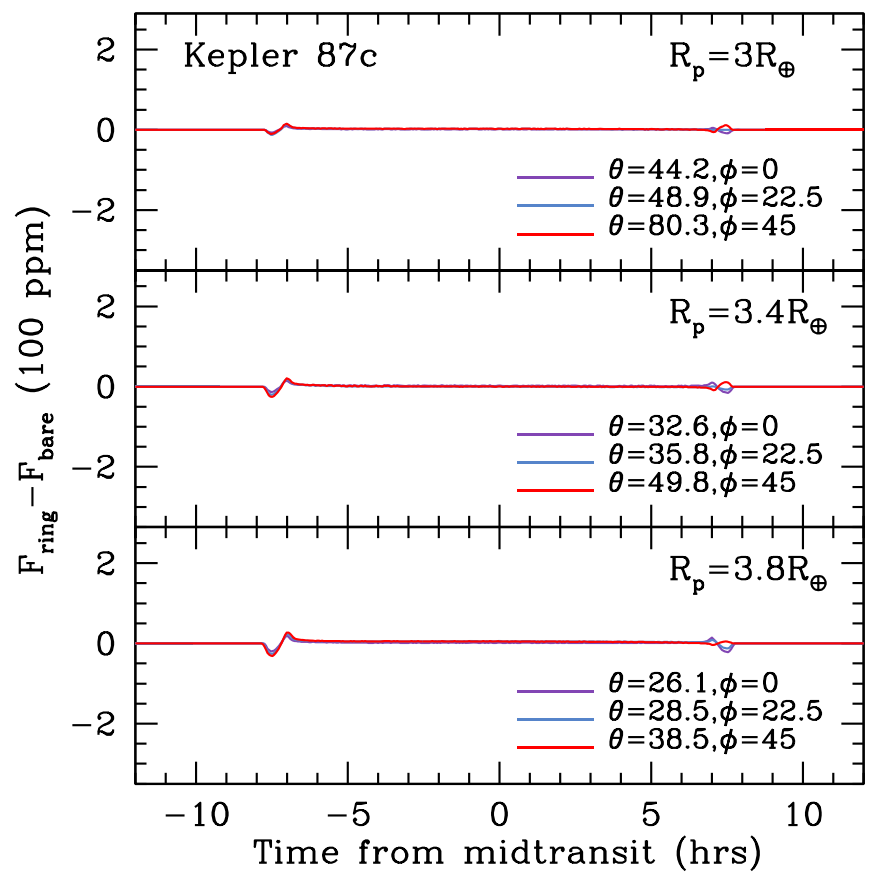

Figure 8. Same as for Figure 6, but for Kepler 87c.

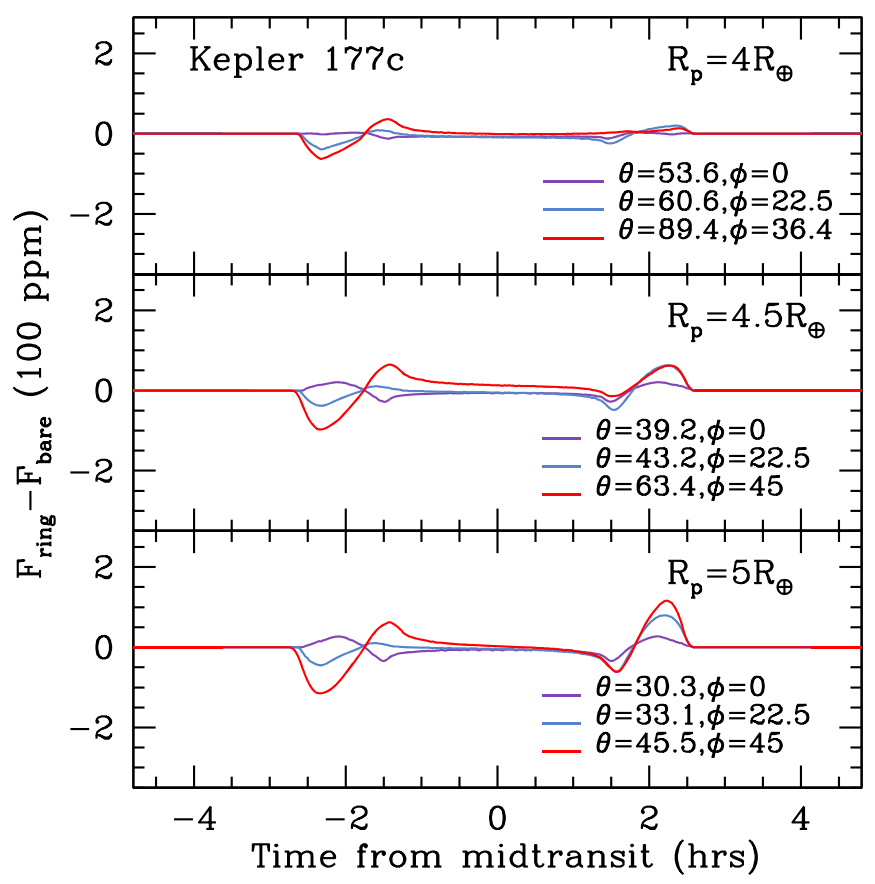

Figure 9. Same as for Figure 6, but for Kepler 177c.

$0.65 \mathrm{~g} \mathrm{~cm}^{-3}$, respectively. Kepler $177 \mathrm{c}$ is more promising because of the larger value of $\left(R_{\mathrm{inf}} / R_{*}\right)^{2}$, making the photometric variations about a factor of $\sim 4$ larger.

\subsection{Practical Considerations}

For the super-puffs that are most favorable for detecting rings (e.g., Kepler $18 \mathrm{~d}$ and $177 \mathrm{c}$ above), measuring the residual signal from a ring requires $\sim 100 \mathrm{ppm}$ photometry on 
$\sim 10$ minute timescales, with shorter-period planets requiring finer temporal sampling. Diffuser-assisted photometry on ground-based telescopes has recently been able to approach this level of precision on brighter targets (Stefansson et al. 2017, 2018; von Essen et al. 2019). Considering the faintness of the super-puff host stars, however, this is a difficult goal. The $J$ magnitudes of the Kepler planets in the super-puff sample range from 12 to 14 , for which the best precision on the necessary timescales is closer to $1000 \mathrm{ppm}$ for diffuserassisted observations on 3-5 m telescopes (Stefansson et al. 2017; Vissapragada et al. 2020). Moving to $10 \mathrm{~m}$ class ground-based facilities would improve the limiting precision, but not by an order of magnitude. Stacking multiple transit observations may also improve the limiting precision, as the ring signal would be effectively static over multiple observations due to the long precession period. However, the difficulty of scheduling many transit observations for planets with long orbital periods and transit durations would realistically necessitate multiyear observing campaigns to build up the requisite baseline, even for the most favorable targets. Observations aimed at detecting rings can thus only be performed with space-based facilities, at least for the Kepler planets in the sample.

Searches for rings in the Kepler sample have been attempted (Aizawa et al. 2018). Although this work included the superpuffs Kepler 18d, 51b, 51d, and 79d, this analysis could only conclude that rings are not necessary to fit the currently available data. The Transiting Exoplanet Survey Satellite (TESS; Ricker et al. 2015) is not optimized to study these faint, long-period systems either. Using the Web TESS Viewing $\mathrm{Tool}^{4}$, the expected $1 \mathrm{hr}$ photometric precision for Kepler 18 is $1020 \mathrm{ppm}$; this does not take into account the difficulty of actually observing the transits due to the month-long TESS Sectors.

In the case of the Hubble Space Telescope (HST), identifying ring signals via space-based photeometry is similarly challenging to the search for exomoons, and HST observations have provided recent photometric evidence for an exomoon candidate around Kepler 1625b (Teachey \& Kipping 2018). Such searches for low-amplitude photometric signals can be compromised by time-correlated systematics, whether instrumental (e.g., Kepler's sudden pixel sensitivity dropout) or astrophysical (e.g., stellar variability) in nature (Jenkins et al. 2010; Kipping et al. 2012, 2015; Christiansen et al. 2013). Additionally, different detrending methodologies seem to deviate at the required $100 \mathrm{ppm}$ level even for HST measurements of Kepler 1625, a star with similar brightness to the Kepler super-puffs studied here (Teachey \& Kipping 2018; Heller et al. 2019; Kreidberg et al. 2019; Teachey et al. 2019).

Thus for secure photometry at the required precision, we must wait for the superior photometry of the James Webb Space Telescope (JWST), even for the most optimistic ring scenarios considered above (Beichman et al. 2014). This adds to the list of predicted explanations for super-puff radii that are testable with JWST; observations with JWST may also be able to detect mid-infrared molecular features above a photochemical haze (Kawashima et al. 2019), as well as diagnostic photometric features of a dusty outflow (Libby-Roberts et al. 2020; Wang \& Dai 2019).

\footnotetext{
4 https://heasarc.gsfc.nasa.gov/cgi-bin/tess/webtess/wtv.py
}

\section{Conclusions and Discussion}

In this work we considered whether the super-puffs, planets with seemingly large radii for their masses, can be explained as ringed. We find that this hypothesis works better for some of the super-puffs and worse for others. Our main conclusions are as follows.

1. The requirement that the rings be composed of rocky material favors Kepler 18d, 87c, 223d, and 223e as possibly being ringed. Kepler $177 \mathrm{c}$ is borderline.

2. The planets must be sufficiently oblate to prevent warping of the rings. This favors Kepler 51c, 51d, 79d, $87 \mathrm{c}$, and $177 \mathrm{c}$, both because of their long synchronization times and the low $J_{2}$ required to prevent warping.

3. Even if rings are present, the planets underlying superpuffs must still have substantial gaseous envelopes. This is supported both by the densities we find for the underlying planets in our simulations and also to make their tidal locking timescales sufficiently long that $J_{2}$ can potentially be large.

4. Taken together, rings likely cannot explain the entire super-puff population, but Kepler $87 \mathrm{c}$ and $177 \mathrm{c}$ have the best chance of being explained by rings. Kepler 18d, 223d, and 223e may also be interesting in case they are spinning faster than what is estimated here. Finally, Kepler $79 \mathrm{~d}$ can only have rings if the ring material is especially porous.

5. Detection of rings via transits will be easiest to test for the higher density $\left(\gtrsim 0.2 \mathrm{~g} \mathrm{~cm}^{-3}\right)$ super-puffs or ones that have a higher overall signal as seen through the ratio $\left(R_{\text {inf }} / R_{p}\right)^{2}$. This favors Kepler $18 \mathrm{~d}, 51 \mathrm{~b}$, and $177 \mathrm{c}$ for testing this hypothesis.

6. Detection of rings will be hardest for planets that have the lower densities $\left(\lesssim 0.1 \mathrm{~g} \mathrm{~cm}^{-3}\right)$ and smaller $\left(R_{\text {inf }} / R_{p}\right)^{2}$, such as Kepler 79d, 87c, 223d, and 223e.

7. Except for HIP 41378f, which is discussed below, current ground- and space-based facilities are not precise enough to test the ring hypothesis. For the Kepler super-puffs, such a test must wait for the launch of JWST.

Confirmation of the presence of rocky rings in some cases would not only be an amazing new discovery, but also provide important information about these planets. This would allow a constraint on the obliquity and the spin through the quadrupole. Both of these would have implications for how the planets migrated to their current location, since even for the ringed hypothesis, it is likely that these planets were formed at larger stellocentric radii and migrated inward (Lee \& Chiang 2016). For rings to provide the necessarily large transits, the planets must maintain a large obliquity through this migration. Recent work shows that this may naturally be expected during the migration of closely packed systems (Millholland \& Laughlin 2019; Millholland 2019), and the detection of rings would allow this affect to be directly measured.

During the finishing stages of this manuscript, HIP $41378 \mathrm{f}$ was announced as a new super-puff (Santerne et al. 2019), which deserves some mention here. Super-puffs have primarily been characterized by transit-timing variations (TTVs; Agol et al. 2005; Holman \& Murray 2005; Agol \& Fabrycky 2018; JontofHutter 2019). For long-period planets, TTVs typically have more success than radial velocity (RV) measurements in identifying low-density planets due to a detection-sensitivity bias effect (Mills \& Mazeh 2017) as well as the intrinsic faintness of most 
super-puff systems. The measurement of the mass of HIP $41378 \mathrm{f}$ via RVs demonstrates that both techniques are capable of exploring super-puffs. This planet is especially exciting concerning the arguments presented here. Its large semimajor axis (1.37 au) makes it less susceptible to having its rings warped, and for the bright parent star $(J=7.98), 100$ ppm photometry is conceivable with current ground- and space-based facilities. The long transit duration and orbital period of HIP 41378f, however, make it a difficult target to schedule for the required phase coverage (global networks of high-precision photometers may alleviate this issue; see von Essen et al. 2018).

We thank Babatunde Akinsanmi, Jason Barnes, Konstantin Batygin, Eve Lee, Heather Knutson, Jessica Libby-Roberts, Alex Teachey, and Johanna Teske for helpful discussions. S.V. is supported by an NSF Graduate Research Fellowship and the Paul \& Daisy Soros Fellowship for New Americans.

\section{ORCID iDs}

Anthony L. Piro (ib https://orcid.org/0000-0001-6806-0673 Shreyas Vissapragada (10 https://orcid.org/0000-00032527-1475

\section{References}

Agol, E., \& Fabrycky, D. C. 2018, in Handbook of Exoplanets, ed. H. J. Deeg \& J. A. Belmonte (Cham: Springer), 7

Agol, E., Steffen, J., Sari, R., \& Clarkson, W. 2005, MNRAS, 359, 567

Aizawa, M., Masuda, K., Kawahara, H., \& Suto, Y. 2018, AJ, 155, 206

Aizawa, M., Uehara, S., Masuda, K., Kawahara, H., \& Suto, Y. 2017, AJ, 153,193

Akinsanmi, B., Barros, S. C. C., Santos, N. C., et al. 2019, A\&A, 621, A117

Akinsanmi, B., Oshagh, M., Santos, N. C., \& Barros, S. C. C. 2018, A\&A, 609, A21

Barnes, J. W., \& Fortney, J. J. 2003, ApJ, 588, 545

Barnes, J. W., \& Fortney, J. J. 2004, ApJ, 616, 1193

Beichman, C., Benneke, B., Knutson, H., et al. 2014, PASP, 126, 1134

Carry, B. 2012, P\&SS, 73, 98

Chandrasekhar, S. 1969, Ellipsoidal Figures of Equilibrium (New Haven, CT: Yale Univ. Press)

Christiansen, J. L., Clarke, B. D., Burke, C. J., et al. 2013, ApJS, 207, 35

Cochran, W. D., Fabrycky, D. C., Torres, G., et al. 2011, ApJS, 197, 7

Daisaka, H., Tanaka, H., \& Ida, S. 2001, Icar, 154, 296

Fabrycky, D. C., Lissauer, J. J., Ragozzine, D., et al. 2014, ApJ, 790, 146

Gaudi, B. S., Chang, H.-Y., \& Han, C. 2003, ApJ, 586, 527

Goldreich, P., \& Tremaine, S. D. 1978, Icar, 34, 240

Hadden, S., \& Lithwick, Y. 2014, ApJ, 787, 80
Hadden, S., \& Lithwick, Y. 2017, AJ, 154, 5

Hedman, M. M. 2015, ApJL, 801, L33

Heising, M. Z., Marcy, G. W., \& Schlichting, H. E. 2015, ApJ, 814, 81

Heller, R., Rodenbeck, K., \& Bruno, G. 2019, A\&A, 624, A95

Holman, M. J., \& Murray, N. W. 2005, Sci, 307, 1288

Jenkins, J. M., Caldwell, D. A., Chandrasekaran, H., et al. 2010, ApJL, 713, L87

Jontof-Hutter, D. 2019, AREPS, 47, 141

Jontof-Hutter, D., Lissauer, J. J., Rowe, J. F., \& Fabrycky, D. C. 2014, ApJ, 785,15

Kawashima, Y., Hu, R., \& Ikoma, M. 2019, ApJL, 876, L5

Kenworthy, M. A., \& Mamajek, E. E. 2015, ApJ, 800, 126

Kipping, D. M., Bakos, G. Á., Buchhave, L., Nesvorný, D., \& Schmitt, A. 2012, ApJ, 750, 115

Kipping, D. M., Huang, X., Nesvorný, D., et al. 2015, ApJL, 799, L14

Kreidberg, L., Luger, R., \& Bedell, M. 2019, ApJL, 877, L15

Lang, K. R. 1992, Astrophysical Data. I. Planets and Stars (New York: Springer)

Lee, E. J. 2019, ApJ, 878, 36

Lee, E. J., \& Chiang, E. 2016, ApJ, 817, 90

Libby-Roberts, J. E., Berta-Thompson, Z. K., Desert, J.-M., et al. 2020, AJ, 159,57

Masuda, K. 2014, ApJ, 783, 53

Miller, N., \& Fortney, J. J. 2011, ApJL, 736, L29

Millholland, S. 2019, ApJ, 886, 72

Millholland, S., \& Laughlin, G. 2019, NatAs, 3, 424

Mills, S. M., Fabrycky, D. C., Migaszewski, C., et al. 2016, Natur, 533, 509

Mills, S. M., \& Mazeh, T. 2017, ApJL, 839, L8

Murray, C. D., \& Dermott, S. F. 1999, Solar System Dynamics (Cambridge: Cambridge Univ. Press)

Ofir, A., Dreizler, S., Zechmeister, M., \& Husser, T.-O. 2014, A\&A, 561, A103

Ohta, Y., Taruya, A., \& Suto, Y. 2009, ApJ, 690, 1

Piro, A. L. 2018a, AJ, 156, 80

Piro, A. L. 2018b, AJ, 156, 54

Ricker, G. R., Winn, J. N., Vanderspek, R., et al. 2015, JATIS, 1, 014003

Rieder, S., \& Kenworthy, M. A. 2016, A\&A, 596, A9

Santerne, A., Malavolta, L., Kosiarek, M. R., et al. 2019, arXiv:1911.07355

Santos, N. C., Martins, J. H. C., Boué, G., et al. 2015, A\&A, 583, A50

Schlichting, H. E., \& Chang, P. 2011, ApJ, 734, 117

Stefansson, G., Li, Y., Mahadevan, S., et al. 2018, AJ, 156, 266

Stefansson, G., Mahadevan, S., Hebb, L., et al. 2017, ApJ, 848, 9

Teachey, A., Kipping, D., Burke, C. J., Angus, R., \& Howard, A. W. 2019, arXiv: 1904.11896

Teachey, A., \& Kipping, D. M. 2018, SciA, 4, eaav1784

Thorngren, D. P., \& Fortney, J. J. 2018, AJ, 155, 214

Tremaine, S., Touma, J., \& Namouni, F. 2009, AJ, 137, 3706

Vissapragada, S., Jontof-Hutter, D., Shporer, A., et al. 2020, AJ, 159, 108

von Essen, C., Ofir, A., Dreizler, S., et al. 2018, A\&A, 615, A79

von Essen, C., Stefansson, G., Mallonn, M., et al. 2019, A\&A, 628, A115

Wang, L., \& Dai, F. 2019, ApJL, 873, L1

Zuluaga, J. I., Kipping, D. M., Sucerquia, M., \& Alvarado, J. A. 2015, ApJL, 803, L14 